\title{
Detecting of All Zero Blocks in HEVC for RDOQ
}

\author{
Nana SHAN ${ }^{\mathrm{a}, 1}$, Henglu WEI ${ }^{\mathrm{b}}$, Wei $\mathrm{ZHOU}^{\mathrm{c}}$ and Zhemin DUAN ${ }^{\mathrm{c}}$ \\ ${ }^{a}$ Taishan University, China \\ ${ }^{\mathrm{b}}$ Tsinghua University, China \\ ${ }^{\mathrm{c}}$ Northwestern Polytechnical University, China
}

\begin{abstract}
There are a larger number of blocks with all zero-quantized coefficient of the transform and quantization process in HEVC. The coding time of transform and quantization can be greatly reduced by skipping all zero coefficient blocks. All zero coefficient blocks detection algorithms for RDOQ are proposed in this paper. The stair-like thresholds are obtained by statistical analysis, which can speed up all zero coefficient blocks detection for RDOQ to improve the coding efficiency. Experimental results show that it can reduce $40 \%$ coding time with negligible loss of BDBR.
\end{abstract}

Keywords. HEVC, all zero blocks, RDO quantization, SATD

\section{Introduction}

High Efficiency Video Coding (HEVC) provides better compression performance than H.264/AVC [1]. Transform or quantization (T/Q) are the most time-consuming parts in HEVC [2]. The basic unit for transform and quantization in HEVC is called transform block (TB). If every quantization level (QL) in a TB is zero, this TB will be called an all zero coefficient blocks (AZB). By avoiding T/Q operation of AZB, the complexity of T/Q can be reduced greatly [3].

Many detection algorithms for all zero coefficient blocks were proposed to reduce the coding complexity. Wang use the sum of absolute difference to detect zero quantized discrete cosine transform [4]. Liu adopt the multiple reference frames to reduce the search range [5]. In [6], Hadamard transform (HT)based method was extended into HEVC, and the upper boundary of sum of absolute difference (SAD) was deduced to reduce the overall complexity. Detecting all zero blocks method was proposed in [7] to speed up the encoding process by categorizing the blocks.

AZB detection algorithm for RDOQ is proposed in this paper. The proposed algorithm is based on the conclusion that a TB is an AZB if the QL of the global maximum magnitude (MM) is zero. By statistical analysis of the MM, the stair-like thresholds for the global MM are obtained. AZBs in RDOQ can be determined by comparing the float QL (FQL) of the estimated coefficients with the stair-like thresholds.

${ }^{1}$ Corresponding author, Nana Shan; E-mail: helensnn@hotmail.com. This work was supported by the Scientific Research Foundation of Taishan University (Grant No. Y-01-2017003). 


\section{Global MM Estimation}

The proposed DCT coefficients estimation algorithm includes two steps. First, MM estimation and mixed granularity coefficients estimation by using zero mean Gaussian distribution model. Then, the standard deviation of DCT coefficients is adopted to estimate the global MM.

The zero mean Gaussian distribution of prediction residuals can be expressed as,

$$
p(x)=\frac{1}{\sqrt{2 \pi} \sigma} e^{-\frac{x^{2}}{2 \sigma^{2}}}
$$

According to (1), the expectation of $|x|$ is,

$$
E[|x|]=\int_{-\infty}^{+\infty}|x| \frac{1}{\sqrt{2 \pi} \sigma} e^{-\frac{x^{2}}{2 \sigma^{2}}} d x=\sqrt{\frac{2}{\pi}} \sigma
$$

where $N$ is the size of TB, and $S A D$ is sum of absolute difference,

$$
S A D=\sum_{x=0}^{N-1} \sum_{y=0}^{N-1}|x|
$$

And we get the global MM:

$$
M M_{e s t}[S A D]=\gamma_{N} \sqrt{\frac{\pi}{2}} \frac{S A D}{N^{2}} \cdot \max _{u, v \in[0, N-1]}\left(\sqrt{\left[D R D^{T}\right]_{u, u}\left[D R D^{T}\right]_{v, v}}\right)
$$

Table 1 shows the probability of the global MM in the selected low frequency coefficients of HEVC standard test sequences range from Class A to Class F. Most of global MMs are located in the selected low frequency positions.

Table 1. The probability of the global MM in the selected low frequency coefficients (\%)

\begin{tabular}{rrrr}
\hline Class & $\mathbf{8} \times \mathbf{8} \mathbf{~ T B}$ & $\mathbf{1 6} \times \mathbf{1 6} \mathbf{~ T B}$ & $\mathbf{3 2} \times \mathbf{3 2} \mathbf{~ T B}$ \\
\hline Class A & 99.16 & 98.14 & 93.12 \\
Class B & 92.66 & 89.95 & 81.91 \\
Class C & 91.77 & 85.36 & 81.44 \\
Class D & 90.37 & 81.33 & 72.80 \\
Class E & 97.40 & 91.82 & 82.29 \\
Class F & 93.85 & 89.75 & 87.96 \\
Average & 94.20 & 89.39 & 83.25 \\
\hline
\end{tabular}

\section{AZB detection in HEVC}

A TB is an AZB when the QL of the global MM is zero. Rather than estimate the RDcost, a new method is proposed in this paper. Then, the next step is to decide the QL of the global MM.

As diagonal QL scan pattern is used for inter prediction coding unit in HEVC, the QL of the global MM is related to the position of coefficients matrix. To explore the relationship between zero QL of the global MM and its location, all FQLs of the global MM less than 1.0 have been collected. The statistical result of $(0,0)$ position of $4 \times 4$ blocks, which means the global MM is located in the $(0,0)$ position of $4 \times 4$ block, is shown in Figure 1 (a). The green line is the number of the FQL quantized to zero QL, and the red line is the number of the FQL quantized to non-zero QL. It can be seen from 
the figure that when the FQL is less than 0.5, the corresponding QL is zero, as the red line equals to zero.

FQL can be used as the threshold to distinct zero QL and non-zero QL of the global MM. Figure 1 (b) shows the relationship between FQL and the ratio of zero QL by using the curve of $p /(p+q)$, where $p$ and $q$ are the number of FQLs quantized to zero QL and non-zero QL respectively. The value of $\mathrm{p} /(\mathrm{p}+\mathrm{q})$ represents the ratio of FQL quantized to zero QL. It can be seen from figure 1 (b) that the probability of the global MM quantized to zero decreases with the increasing of FQL. When $p /(p+q)$ equals to 0.9 , the value of FQL is selected as the threshold between zero QL and non-zero QL, which is 0.61 . If the global MM is located in $(0,0)$ position of a $4 \times 4$ block, and its FQL is less than 0.61 , the corresponding QL is considered as zero.

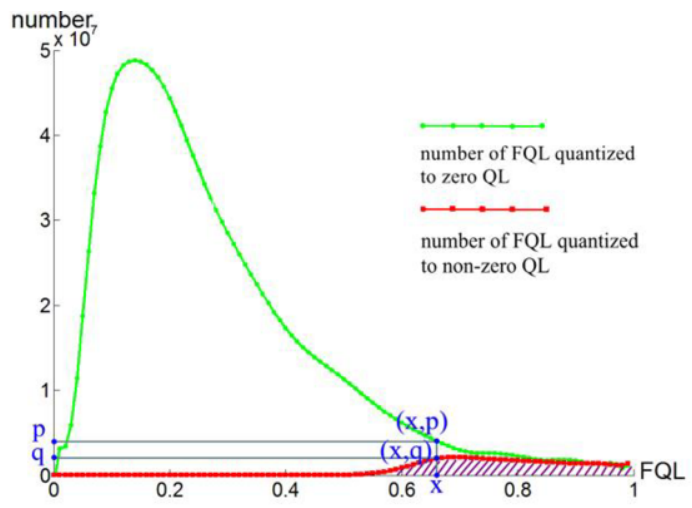

(a) Number of FQL

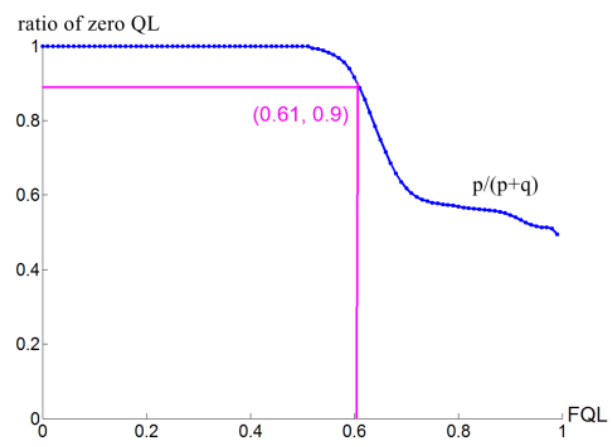

(b) The ratio of FQL quantized to zero QL

Figure 1. The collection result of the FQLs in $(0,0)$ position of $4 \times 4$ block.

Thresholds for all positions in various TB sizes are shown in figure 2 (b) (e). The threshold is related to the frequency. The main reason is that high frequency coefficients tend to consume more coding bits and they are more likely to be quantized to zero by RDOQ. The above method to detect AZB for DCT can be directly extended to transform skip mode. Thresholds for transform skip mode are shown in figure 2 (a). If the FQL of 
every prediction residual is less than the corresponding threshold, the TB is considered as an AZB in transform skip mode.
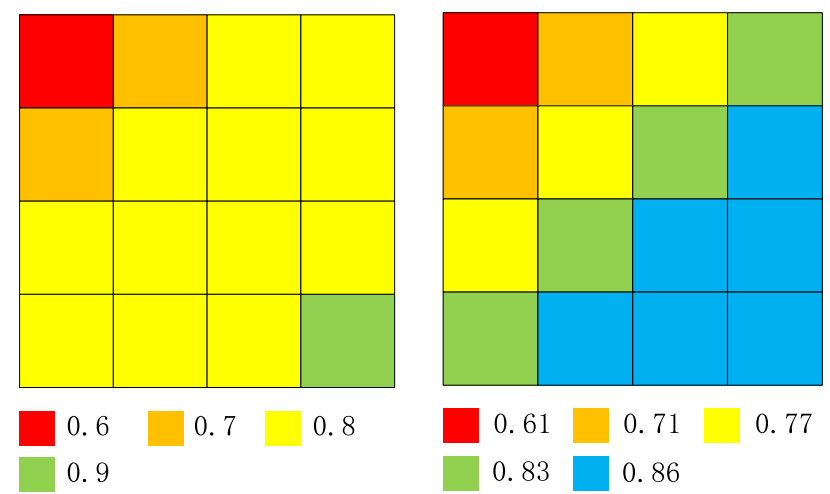

(a) Thresholds for transform skip

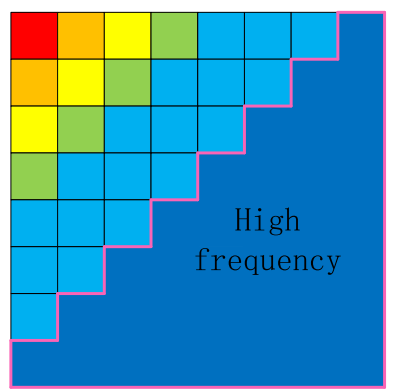

(b) Thresholds for $4 \times 4 \mathrm{~TB}$
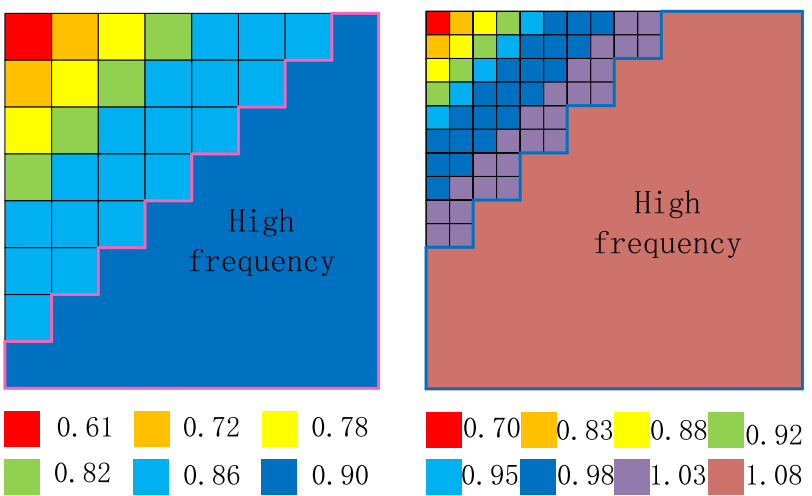

(c) Thresholds for $8 \times 8 \mathrm{~TB}$

(d) Thresholds for $16 \times 16 \mathrm{~TB}$

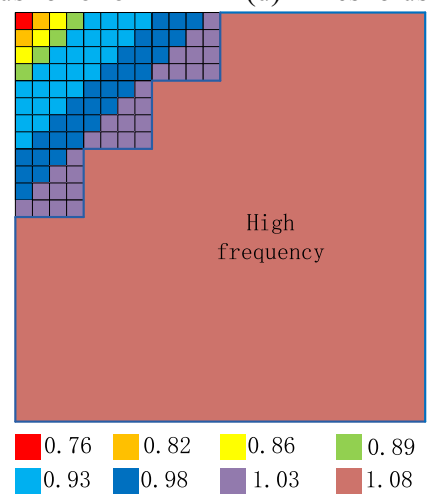

(e) thresholds for $32 \times 32 \mathrm{~TB}$

Figure 2. Thresholds for AZB detection in RDOQ

The method to pre-detect AZBs and non-AZBs by the estimated global MM can be used in RDOQ. The flowchart of AZB detection for DCT in RDOQ is shown in figure 3. The thresholds to detect zero QL in RDOQ vary with the position of coefficients. Firstly, 
the global MM is estimated to pre-detect AZB and non-AZB. Then, low frequency coefficients are used. Lastly, the MM of high frequency coefficients is estimated to determine AZB and non-AZB.

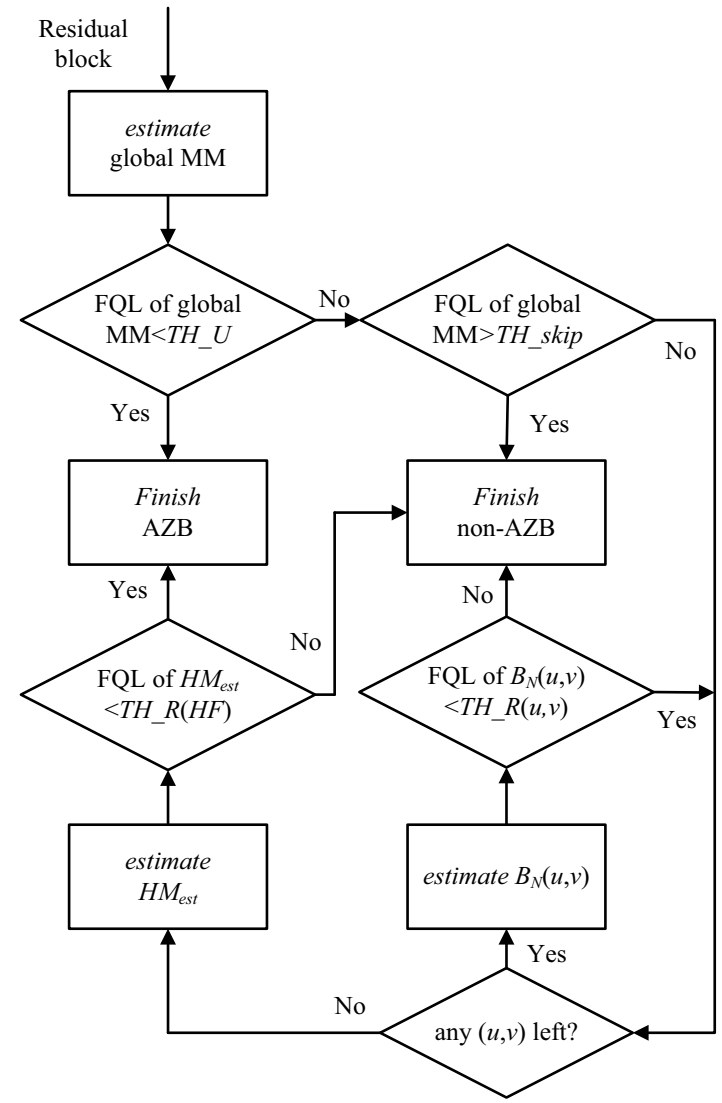

Figure 3. The flowchart of AZB detection in RDOQ

\section{Experimental results}

The accuracy of AZB detection process can be measured by false acceptance ration (FAR) and false rejection ratio (FRR). They can be calculated as equation (5),

$$
\begin{aligned}
& F R R=\frac{\text { num }_{m z}}{\text { num }_{z}} \\
& F A R=\frac{\text { num }_{m n}}{\text { num }_{n}}
\end{aligned}
$$

The num $_{m z}$ means the number of AZB which are classified as non-AZB incorrectly,

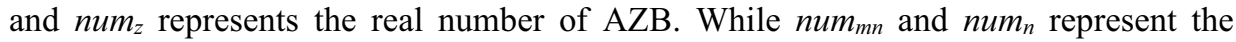
number of non-AZB which is misclassified as AZB and the real number of non-AZB. FRR means the ratio of $A Z B$ which are classified as non-AZB incorrectly, while FAR means the ratio of non-AZB misclassified as AZB. We want to detect AZB as much as 
possible, which means that the FRR and FAR will be very low. In other words, higher detection efficiency means lower FRR and FAR.

The results of the proposed algorithm are shown as Table 2 . The average FRR of $4 \times 4,8 \times 8,16 \times 16$ and $32 \times 32$ TBs are $8.2 \%, 20.0 \%, 21.0 \%$ and $39.3 \%$ respectively. The average FAR of $4 \times 4,8 \times 8,16 \times 16$ and $32 \times 32$ TBs are $2.0 \%, 1.7 \%, 3.9 \%$ and $2.1 \%$ respectively. From Table 2, the proposed method is better than Lee [8] and Fan [9].

Table 2. The Results for FRR and FAR

\begin{tabular}{|c|c|c|c|c|c|c|c|}
\hline \multirow[t]{2}{*}{ Sequence } & \multirow[t]{2}{*}{ Sequence } & \multicolumn{2}{|c|}{ Lee $[8]$} & \multicolumn{3}{|c|}{ Fan [9] } & \multirow{2}{*}{$\begin{array}{r}\text { Proposed } \\
\text { FAR } \\
(\%) \\
\end{array}$} \\
\hline & & $\begin{array}{r}\text { FRR } \\
(\%)\end{array}$ & $\begin{array}{r}\text { FAR } \\
(\%)\end{array}$ & $\begin{array}{r}\text { FRR } \\
(\%)\end{array}$ & $\begin{array}{r}\text { FAR } \\
(\%)\end{array}$ & $\begin{array}{r}\text { FRR } \\
(\%)\end{array}$ & \\
\hline \multirow[t]{4}{*}{$480 \mathrm{P}$} & $4 \times 4$ & 9.7 & 2.0 & 3.7 & 7.8 & 6.65 & 2.83 \\
\hline & $8 \times 8$ & 22.5 & 1.6 & 10.0 & 16.5 & 16.48 & 2.28 \\
\hline & $16 \times 16$ & 21.2 & 9.4 & 15.4 & 16.0 & 17.73 & 5.07 \\
\hline & $32 \times 32$ & 52.5 & 2.6 & 28.3 & 12.5 & 34.15 & 2.77 \\
\hline \multirow[t]{4}{*}{$1080 \mathrm{P}$} & $4 \times 4$ & 8.9 & 2.2 & 2.7 & 9.9 & 9.77 & 1.24 \\
\hline & $8 \times 8$ & 22.5 & 2.9 & 16.3 & 19.0 & 23.53 & 1.02 \\
\hline & $16 \times 16$ & 13.7 & 21.6 & 11.4 & 20.0 & 24.19 & 2.70 \\
\hline & $32 \times 32$ & 40.1 & 8.4 & 30.1 & 16.3 & 44.42 & 1.35 \\
\hline \multirow[t]{4}{*}{ Average } & $4 \times 4$ & 9.3 & 2.1 & 3.2 & 8.9 & 8.2 & 2.0 \\
\hline & $8 \times 8$ & 22.5 & 2.3 & 13.2 & 17.8 & 20.0 & 1.7 \\
\hline & $16 \times 16$ & 17.5 & 15.5 & 13.4 & 18.0 & 21.0 & 3.9 \\
\hline & $32 \times 32$ & 46.3 & 5.5 & 29.2 & 14.4 & 39.3 & 2.1 \\
\hline
\end{tabular}

The following experimental results are based on HEVC test model version 13.0 (HM 13.0). The standard encoder_lowdelay_main.cfg configuration file is used. 21 typical test sequences are selected from Class A to Class F, with the quantization parameters (QP) set to 22, 27, 32, and 37 respectively. Comparing with the performance of Lee [8] and Fan [9], the change of BDBR [10] and coding time are shown on Table 3. As Table 3 shows the average time saving of the proposed method is $40 \%$, while the number of Lee [8] and Fan [9] is 34\% and 43\%. For BDBR, the proposed algorithm is better than Lee [8] and Fan [9].

Table 3. The Results for RDOQ AZB Detection

\begin{tabular}{rrrrrrr}
\hline Sequence & \multicolumn{2}{c}{ Lee [8] } & & Fan [9] & Proposed \\
\cline { 2 - 7 } & $\begin{array}{r}\text { BDBR } \\
\mathbf{( \% )}\end{array}$ & $\begin{array}{r}\text { TS } \\
\mathbf{( \% )}\end{array}$ & $\begin{array}{r}\text { BDBR } \\
\mathbf{( \% )}\end{array}$ & $\begin{array}{r}\text { TS } \\
\mathbf{( \% )}\end{array}$ & $\begin{array}{r}\text { BDBR } \\
\mathbf{( \% )}\end{array}$ & $\begin{array}{r}\text { TS } \\
\mathbf{( \% )}\end{array}$ \\
\hline PeopleOnStreet & 0.60 & 22 & 0.61 & 25 & 0.11 & 35 \\
Traffic & 0.57 & 41 & 0.94 & 50 & 0.20 & 39 \\
BasketballDrive & 0.47 & 32 & 0.76 & 38 & 0.31 & 34 \\
BQTerrace & 0.44 & 43 & 0.70 & 54 & 0.03 & 44 \\
Cactus & 0.31 & 34 & 0.90 & 44 & -0.09 & 35 \\
Kimono & -0.02 & 11 & 0.02 & 10 & -0.11 & 34 \\
ParkScene & 0.55 & 40 & 0.89 & 46 & 0.27 & 39 \\
BasketballDrill & 0.24 & 34 & 0.62 & 48 & 0.00 & 33 \\
BQMall & 0.75 & 37 & 1.00 & 53 & 0.22 & 33 \\
PartyScene & 0.83 & 34 & 0.80 & 41 & 0.10 & 26 \\
RaceHorsesC & 0.62 & 20 & 0.43 & 34 & -0.07 & 21 \\
BasketballPass & 0.86 & 36 & 0.80 & 45 & 0.13 & 31 \\
BlowingBubbles & 0.68 & 29 & 0.79 & 38 & 0.20 & 29 \\
BQSquare & 0.80 & 38 & 0.48 & 50 & 0.22 & 36 \\
RaceHorsesD & 0.41 & 18 & 0.34 & 20 & 0.19 & 20 \\
FourPeople & 0.30 & 53 & 0.36 & 64 & 0.16 & 59 \\
Johnny & 0.57 & 53 & 0.46 & 65 & 0.48 & 63 \\
KristenAndSara & 0.26 & 48 & 0.33 & 58 & 0.10 & 61
\end{tabular}




\begin{tabular}{rrrrrrr} 
ChinaSpeed & 0.46 & 23 & 0.46 & 30 & 0.08 & 31 \\
SlideEditing & 0.41 & 57 & 0.20 & 69 & 0.14 & 70 \\
SlideShow & 0.42 & 21 & 1.01 & 19 & 0.25 & 58 \\
Average & 0.50 & 34 & 0.61 & 43 & 0.14 & 40 \\
\hline
\end{tabular}

\section{Conclusion}

An AZB detection algorithm for RDOQ is proposed in this paper. The algorithm adopts a global MM strategy based on low and high frequency coefficients to estimate transform coefficients. The proposed algorithms are tested on HEVC test model to compare the results with other methods. Experiment results show that the proposed algorithm can efficiently reduce the computation complexity while keeping nearly the same RD performance with the original algorithm in HEVC.

\section{References}

[1] Ohm, J., Sullivan, G. J., Schwarz, H., Tan, T. K., \& Wiegand, T. Comparison of the coding efficiency of video coding standards - including high efficiency video coding (hevc). Circuits \& Systems for Video Technology IEEE Transactions on, 22(12), 2012, 1669 - 1684.

[2] Bossen, F., Bross, B., Suhring, K., \& Flynn, D. Hevc complexity and implementation analysis. Circuits \& Systems for Video Technology IEEE Transactions on, 22(12), 2012, 1685 - 1696.

[3] Jung, S. W., Baek, S. J., Park, C. S., \& Ko, S. J. Fast mode decision using all-zero block detection for fidelity and spatial scalable video coding. IEEE Transactions on Circuits \& Systems for Video Technology, 20(2), 2010, 201-206.

[4] Wang, H., \& Kwong, S. Prediction of zero quantized det coefficients in h.264/avc using hadamard transformed information. IEEE Transactions on Circuits \& Systems for Video Technology, 18(4), 2008, $510-515$.

[5] Liu, Z., Li, L., Song, Y., Li, S., Goto, S., \& Ikenaga, T. Motion feature and hadamard coefficient-based fast multiple reference frame motion estimation for h.264. IEEE Transactions on Circuits \& Systems for Video Technology, 18(5), 2008, 620-632.

[6] Sole, J., Joshi, R., Nguyen,, Ji, T., Karczewicz, M., \& Clare, G., et al. Transform coefficient coding in hevc. Circuits \& Systems for Video Technology IEEE Transactions on, 22(12), 2012, 1765-1777.

[7] Wei H, Wei Z, Zhang X, et al. All zero block detection for HEVC based on the quantization level of the maximum transform coefficient. Multimedia Tools and Applications, 2018, 78.

[8] Lee, K., Lee, H. J., Kim, J., \& Choi, Y. A novel algorithm for zero block detection in high efficiency video coding. IEEE Journal of Selected Topics in Signal Processing, 7(7), 2013, 1124-1134.

[9] Fan, H., Wang, R., Ding, L., Xie, X., Jia, H., \& Gao, W. Hybrid Zero Block Detection for High Efficiency Video Coding. IEEE Transactions on Multimedia, 18(3), 2016,537-543.

[10] Bjontegaard, G. Calculation of average psnr difference between rd-curves. ITU-T VCEG-M33, April, 2001. 